\title{
Providing safe and timely patient-centred care
}

\author{
Authors: Beth Griffiths, Firdaus Adenwalla and Annette Davies
}

\section{Aims}

This abstract describes how a small team of nurses (supported by a consultant physician) provides safe, timely, evidence-based efficient care to their patients that would have traditionally been done in a hospital setting.

\section{Methods}

The Acute Clinical Team (ACT) works as part of the multidisciplinary Neath Port Talbot Community Resource Team in a Welsh valley. It is an advanced nurse practitioner (ANP)-led service that is supported by a consultant physician/geriatrician. The team provides rapid assessment and clinical interventions in the person's place of residence (private home or care home), which is responsive and reflexive. They deliver holistic clinical interventions addressing problems that would traditionally have required a hospital admission or a longer hospital stay.

This service has grown over the past 12 years: relationships with GPs, other community teams and secondary care services have gone from strength to strength and referral numbers have increased year on year. In the 6 months from January 2017 to June 2017, the team looked after 570 patients. The most common problems encountered were dehydration, respiratory infections, falls, anaemia, cellulitis, urinary tract infection, exacerbation of heart failure, acute kidney injury, fast atrial fibrillation and general frailty. Treatment examples include intravenous (IV) antibiotics, IV fluids / subcutaneous fluids (dehydration), pain management, treatment for fast atrial fibrillation and titrating heart failure medication.

\section{Results}

Prevention of emergency hospital admissions is the primary aim for this team. According to the National Wales Information Service, unscheduled hospital admissions for persons over the age of 80 years reduced by $14 \%$ in the Neath Port Talbot catchment area (2010-2016). Satisfaction ratings for patients, carers, referrers and staff working in the service are all very good. These are regularly reviewed to ensure that quality underpins the service.

Authors: Neath Port Talbot Community Resource Team, Abertawe Bro Morgannwg University Health Board, Swansea, UK

\section{Conclusion}

A number of innovations and developments help to keep this team at the forefront of quality care delivery. Some of these include:

$>$ taking direct referrals from nursing homes, the ambulance control desk and the learning disability team

> using nursing home beds for step-up care when a hospital admission is not absolutely necessary

> using iPads for electronic diaries, electronic prescribing, accessing patient records and escalation of problems

> point-of-care testing: blood tests, blood gases, urinalysis, electrocardiogram (ECG), bladder scanning and midline insertion.

Working in this way is changing the culture with patients, families and referrers, recognising that care in the community is safe and a viable option to hospital admission.

\section{Conflict of interest statement}

There are no conflicts of interest to declare. 